\title{
WEAKLY CLOSED $m$-ACCRETIVE OPERATORS ${ }^{1}$
}

\author{
A. YAMAMOTO AND N. OKAZAWA
}

\begin{abstract}
Let $A$ and $B$ be weakly closed, nonlinear $m$-accretive (single-valued) operators in a reflexive Banach space $X$, and $B_{n}$ be the Yosida approximation of $B$. Then the following condition is sufficient for the sum $A+B$ to be also $m$-accretive: For each $v \in X,\left\|B_{n} u_{n}\right\|$ is bounded as $n$ tends to infinity, where $u_{n}$ is defined by the equation $u_{n}+A u_{n}+B_{n} u_{n}=v$, $n=1,2, \ldots$ Some related conditions are also provided.
\end{abstract}

1. Introduction. Let $A$ and $B$ be nonlinear (i.e. not necessarily linear) $m$-accretive operators in a Banach space $X$. Then the conditions under which the sum $A+B$ is also $m$-accretive have been studied by several authors (see e.g. [1], [2], [4], [6], [7]). In particular, Fitzgibbon [4] gave such a sufficient condition when $X$ is reflexive and $A, B$ are single-valued and weakly closed. His result is related to a theorem in Kato [6], i.e., it is established by showing that for each $x \in D(A+B)$ there exists a unique solution to the Cauchy problem: $u^{\prime}(t)+(A+B) u(t)=0$ for a.e. $t \geqslant 0, u(0)=x$.

In this note we shall prove a similar theorem, under a slightly generalized assumption, without using such techniques (see Theorem 2.4 and Corollary 2.5 below).

Throughout this note $X$ denotes a reflexive Banach space, with the adjoint space $X^{*}$. The pairing between $u \in X$ and $f \in X^{*}$ is denoted by $(u, f)$. A single-valued operator $A$ (with domain $D(A)$ and range $R(A)$ ) in $X$ is said to be accretive if $\|(u+\lambda A u)-(v+\lambda A v)\| \geqslant\|u-v\|$ for all $u, v \in D(A)$ and $\lambda>0$. Let $F$ be the duality map from $X$ to $X^{*}$ : for each $w \in X, F(w)=\{f$ $\left.\in X^{*} ;(w, f)=\|w\|^{2}=\|f\|^{2}\right\}$. Then an operator $A$ in $X$ is accretive if and only if for all $u, v \in D(A)$ there is $f \in F(u-v)$ such that $\operatorname{Re}(A u-A v, f)$ $\geqslant 0$; see [5]. An accretive operator is said to be $m$-accretive if $R(1+\lambda A)=X$ for some (and hence for all) $\lambda>0$.

2. The main result. Let $A$ and $B$ be $m$-accretive operators in $X$ and $B_{n}$ be the Yosida approximation of $B: B_{n}=n-n\left(1+n^{-1} B\right)^{-1}$. Then $B_{n}$ is everywhere defined and $m$-accretive. Since $B_{n}$ is Lipschitz continuous, $A+B_{n}$ defined on $D(A)$ is also $m$-accretive. Consequently, for each $v \in X$ there exists $u_{n} \in D(A)$ such that

$$
u_{n}+A u_{n}+B_{n} u_{n}=v, \quad n=1,2, \ldots
$$

Received by the editors November 10, 1976 and, in revised form, January 24, 1977.

AMS (MOS) subject classifications (1970). Primary 47H15; Secondary 47B44, 47D05.

Key words and phrases. Nonlinear accretive operator, weak closedness, Yosida approximation, perturbation.

'The authors would like to thank the referee for his kind advice.

(c) American Mathematical Society 1977 
The following lemma is a nonlinear version of Lemma 2.1 in [10].

LEMMA 2.1. Let $A$ be a weakly closed, nonlinear $m$-accretive operator in a reflexive Banach space $X$, and $A_{n}$ be its Yosida approximation.

(i) Let $\left\{u_{n}\right\}$ be a sequence in $D(A)$ such that $u_{n} \rightarrow u \in X(\rightarrow$ denotes weak convergence). If the $\left\|A u_{n}\right\|$ are bounded, then $u \in D(A)$ and $A u_{n} \rightarrow A u$.

(ii) Let $\left\{w_{n}\right\}$ be a sequence in $X$ such that $w_{n} \rightarrow u \in X$. If the $\left\|A_{n} w_{n}\right\|$ are bounded, then $u \in D(A)$ and $A_{n} w_{n} \rightarrow A u$.

Proposition 2.2. Let $X$ be reflexive, and $A$ and $B$ be weakly closed, nonlinear $m$-accretive operators in $X$. Let $u_{n}$ be as in (1). If $\left\|B_{n} u_{n}\right\|$ is bounded as $n$ tends to infinity, then $v \in R(1+A+B)$.

Proof. Since $u_{n}$ is defined by (1), we have

$$
\begin{aligned}
\left\|u_{n}\right\| & \leqslant\left\|u_{n}-u_{1}\right\|+\left\|u_{1}\right\| \leqslant\left\|(1+A) u_{n}-(1+A) u_{1}\right\|+\left\|u_{1}\right\| \\
& \leqslant\left\|B_{n} u_{n}\right\|+\left\|B_{1} u_{1}\right\|+\left\|u_{1}\right\|, \quad n \geqslant 1 .
\end{aligned}
$$

Therefore, $\left\|u_{n}\right\|$ is bounded by assumption. Since $X$ is reflexive, there is a subsequence $\left\{u_{m}\right\}$ of $\left\{u_{n}\right\}$ such that $u_{m} \rightarrow u \in X$. Noting that both $\left\|A u_{m}\right\|$ and $\left\|B_{m} u_{m}\right\|$ are bounded in $m$ by assumption, we see from Lemma 2.1 that $u \in D(A+B), A u_{m} \rightarrow A u$ and $B_{m} u_{m} \rightarrow B u$. Thus we obtain

$$
v=(1+A+B) u \text {. }
$$

Q.E.D.

The next lemma, which generalizes a result in [4], is due to the referee.

LEMMA 2.3. Let $A$ and $B$ be nonlinear accretive operators in a reflexive Banach space $X$. If $A$ is $m$-accretive, then $A+B$ is accretive.

Proof. Let $u, v \in D(A)$. Noting that $A$ is single-valued, we have that $\operatorname{Re}(A u-A v, f) \geqslant 0$ for all $f \in F(u-v)$ (see Miyadera [8, Theorem 3(i')]). Since $B$ is accretive, this yields $\operatorname{Re}((A+B) u-(A+B) v, g) \geqslant 0$ for some $g \in F(u-v)$, whence $A+B$ is accretive. Q.E.D.

Our result is given by

THEOREM 2.4. Let $X$ be reflexive, and $A$ and $B$ be weakly closed, nonlinear $m$-accretive operators in $X$. Let $u_{n}$ be as in (1). If for each $v \in X,\left\|B_{n} u_{n}\right\|$ is bounded as $n$ tends to infinity, then $A+B$ is also $m$-accretive.

Proof. It suffices by Lemma 2.3 to show that $R(1+A+B)=X$. But this follows immediately from assumption and Proposition 2.2. Q.E.D.

The following corollary was first proved by Fitzgibbon [4].

Corollary 2.5. Let $X$ be reflexive, and $A$ and $B$ be weakly closed, nonlinear $m$-accretive operators in $X$, with $D(A) \subset D(B)$. Assume that there exist constants $a \geqslant 0,0 \leqslant b<1$ and $c \geqslant 0$ such that for all $u \in D(A)$,

$$
\|B u\| \leqslant a\|u\|+b\|A u\|+c .
$$

Then $A+B$ is also m-accretive.

Proof. Let $u_{n}$ be as in (1). Then it suffices to show that (2) implies the boundedness of $\left\|B_{n} u_{n}\right\|$ in $n$. Now it follows that 


$$
\begin{aligned}
\left\|B_{n} u_{n}\right\| & \leqslant\left\|B u_{n}\right\| \leqslant a\left\|u_{n}\right\|+b\left\|A u_{n}\right\|+c \\
& \leqslant a\left\|u_{n}\right\|+b\left\|\left(1+A+B_{n}\right) u_{n}\right\|+b\left\|\left(1+B_{n}\right) u_{n}\right\|+c .
\end{aligned}
$$

Therefore, we obtain

$$
(1-b)\left\|B_{n} u_{n}\right\| \leqslant b\|v\|+(a+b)\left\|u_{n}\right\|+c .
$$

Thus, it remains to show that $\left\|u_{n}\right\|$ is bounded in $n$. Let $u \in D(A)$ be fixed. Then $\left\|u_{n}-u\right\| \leqslant\left\|v-\left(1+A+B_{n}\right) u\right\|$. So we obtain

$$
\left\|u_{n}\right\| \leqslant\|v\|+2\|u\|+\|A u\|+\|B u\| \text {. }
$$

Q.E.D.

The next theorem is related to a theorem obtained by Barbu [2].

Theorem 2.6. Let $X$ be reflexive, and $A$ and $B$ be $m$-accretive. Let $A$ be linear, and $B$ be nonlinear and weakly closed. Assume that $D(A)$ is invariant under $\left(1+n^{-1} B\right)^{-1}, n \geqslant 1$, and there exists a constant $c \geqslant 0$ such that for all $u \in D(A)$,

$$
\left\|A\left(1+n^{-1} B\right)^{-1} u\right\| \leqslant\|A u\|+c n^{-1}\|u\|, \quad n \geqslant 1 .
$$

Then the closure of $A+B$ is also $m$-accretive.

Proof. $A+B$ is accretive by Lemma 2.3. Let $\overline{A+B}$ be the closure of $A+B$. Then $\overline{A+B}$ is also accretive. Since $R(1+\overline{A+B})$ is closed, it suffices to show that $R(1+A+B)$ is dense in $X$.

Let $u_{n}$ be as in (1). Since (1) can be written

$$
(A+n+1) u_{n}=v+n\left(1+n^{-1} B\right)^{-1} u_{n}
$$

we have, for $v \in D(A)$,

$$
A u_{n}=(A+n+1)^{-1}\left(A v+n A\left(1+n^{-1} B\right)^{-1} u_{n}\right)
$$

So we obtain, by (3),

$$
\left\|A u_{n}\right\| \leqslant(n+1)^{-1}\|A v\|+(n+1)^{-1} n\left(\left\|A u_{n}\right\|+c n^{-1}\left\|u_{n}\right\|\right) .
$$

It follows that $\left\|A u_{n}\right\| \leqslant\|A v\|+c\left\|u_{n}\right\|$ and hence $\left\|B_{n} u_{n}\right\| \leqslant\|A v\|+\|v\|+$ $(1+c)\left\|u_{n}\right\|$. Now let $u \in D(A)$. Then $(1+B)^{-1} u \in D(A+B)$ and, in the same way as in the proof of Corollary 2.5 , we can prove that $\left\|u_{n}\right\|$ is bounded in $n$. Therefore, by Proposition 2.2 we see that $D(A) \subset R(1+A+B)$. Since $D(A)$ is dense in $X$ (see [11, p. 218]), so is $R(1+A+B)$. Q.E.D.

3. Remark. The purpose of this section is to give a semilinear version of a theorem obtained in [3] and [9].

Proposition 3.1. Let $X$ be reflexive, and $A$ and $B$ be $m$-accretive operators in $X$, with $D(A) \subset D(B)$. Let $A$ be linear and $B$ be nonlinear and weakly closed. Assume that there exist constants $a \geqslant 0, c \geqslant 0$ such that for all $u \in$ $D(A)$,

$$
\|B u\| \leqslant a\|u\|+\|A u\|+c .
$$

If $\overline{A+B}$ is weakly closed, then $\overline{A+B}$ is $m$-accretive. 
Proof. As noted in the proof of Theorem 2.6, $\overline{A+B}$ is accretive. So it suffices to show that $R(1+\overline{A+B})=X$.

Since $\|B u\| \leqslant a\|u\|+t^{-1}\|t A u\|+c$ for $1<t \leqslant 2$, it follows from Corollary 2.5 that $t A+B$ is $m$-accretive. Consequently, for each $w \in X$ there exists a family $\{u(t)\}$ such that

$$
(1+t A+B) u(t)=w, \quad 1<t \leqslant 2 .
$$

As in the proof of Corollary $2.5,\|u(t)\|$ is bounded in $t$. Since $X$ is reflexive, we can find a sequence $\left\{t_{n}\right\}, 1<t_{n} \leqslant 2$, and an element $u_{0} \in X$ such that $t_{n} \rightarrow 1$ and

$$
u\left(t_{n}\right) \rightarrow u_{0} \text { as } n \rightarrow \infty
$$

Next we set

$$
v(t)=(1+A+B) u(t) .
$$

It follows from (5) and (7) that

$$
v(t)-w=(1-t) A u(t), \quad 1<t \leqslant 2 .
$$

By virtue of (4) we have

$$
\begin{aligned}
\|v(t)-w\| & =(t-1)\|A u(t)\| \\
& \leqslant t\|A u(t)\|-(\|B u(t)\|-a\|u(t)\|-c) \\
& \leqslant\|(t A+B) u(t)\|+a\|u(t)\|+c \\
& \leqslant\|w\|+(1+a)\|u(t)\|+c
\end{aligned}
$$

Consequently, $\left\{v\left(t_{n}\right)\right\}$ is a bounded sequence in $X$. We shall show that $v\left(t_{n}\right) \rightarrow w$ as $n \rightarrow \infty$. To this end, let $A^{*}$ be the adjoint of $A$. Then we see from (8) that for $g \in D\left(A^{*}\right)$,

$$
\begin{aligned}
\left|\left(v\left(t_{n}\right)-w, g\right)\right| & =\left|\left(1-t_{n}\right)\left(A u\left(t_{n}\right), g\right)\right| \\
& =\left(t_{n}-1\right)\left|\left(u\left(t_{n}\right), A^{*} g\right)\right| \\
& \leqslant\left(t_{n}-1\right)\left\|u\left(t_{n}\right)\right\|\left\|A^{*} g\right\| \rightarrow 0 \quad(n \rightarrow \infty) .
\end{aligned}
$$

Since $D\left(A^{*}\right)$ is dense in $X^{*}$, it follows that $v\left(t_{n}\right) \rightarrow w$. In view of (6) and (7), we obtain from the weak closedness of $\overline{A+B}, u_{0} \in D(\overline{A+B})$ and $(1+\overline{A+B}) u_{0}=w$. Since $w \in X$ is arbitrary, $R(1+\overline{A+B})=X$. Q.E.D.

\section{REFERENCES}

1. H. Brezis, M. G. Crandall and A. Pazy, Perturbations of nonlinear maximal monotone sets in Banach space, Comm. Pure Appl. Math. 23 (1970), 123-144. MR 41 \#2454.

2. V. Barbu, Weak solutions for nonlinear functional equations in Banach spaces, Ann. Mat. Pura Appl. 87 (1970), 87-110. MR 45 \# 7536.

3. P. R. Chernoff, Perturbations of dissipative operators with relative bound one, Proc. Amer. Math. Soc. 33 (1972), 72-74. MR 45 \# 5804.

4. W. E. Fitzgibbon, Nonlinear perturbation of $m$-accretive operators, Proc. Amer. Math. Soc. 44 (1974), 359-364. MR 49 \# 7856.

5. T. Kato, Nonlinear semigroups and evolution equations, J. Math. Soc. Japan 19 (1967), 508-520. MR 37 \# 1820.

6. Accretive operators and nonlinear evolution equations in Banach spaces, Proc. 
Sympos. Pure Math., vol. 18, part 1, Amer. Math. Soc., Providence, R. I., 1970, pp. 138-161. MR 42 \#6663.

7. Y. Konishi, A remark on perturbation of $m$-accretive operators in Banach space, Proc. Japan Acad. 47 (1971), 452-455. MR 47 \#7540.

8. I. Miyadera, Some remarks on semi-groups of nonlinear operators, Tôhoku Math. J. 23 (1971), 245-258. MR 45 \# 5805.

9. N. Okazawa, A perturbation theorem for linear contraction semigroups on reflexive Banach spaces, Proc. Japan Acad. 47 (1971), 947-949. MR 47 \#2429.

10.

Perturbations of linear m-accretive operators, Proc. Amer. Math. Soc. 37 (1973), 169-174. MR 47 \# 2403.

11. K. Yosida, Functional analysis, 2nd ed., Grundlehren math. Wiss., Band 123, SpringerVerlag, Berlin and New York, 1968. MR 39 \#741.

Aihara Senior High School, Kanagawa Prefecture, Japan

DePartiment of MATHEMatics, Science University of Tokyo, Tokyo 162, JAPAN 